\title{
SISTEMA DE INFORMAÇÕES GEOGRÁFICAS PARA A GESTÃO DE PROGRAMAS MUNICIPAIS DE CUIDADO A IDOSOS ${ }^{1}$ THE USE OF GEOGRAPHIC INFORMATION SYSTEMS FOR MANAGING MUNICIPAL ELDERLY CARE PROGRAMS SISTEMA DE INFORMACIONES GEOGRÁFICAS PARA LA GESTIÓN DE PROGRAMAS MUNICIPALES DE CUIDADO A ANCIANOS
}

\author{
Sofia Cristina Iost Pavarini², Eduardo Mario Mendiondo ${ }^{3}$, Marcelo Montaño ${ }^{4}$, Diogo Martino Fernandes \\ Almeida ${ }^{5}$, Marisa Silvana Zazzetta de Mendiondo ${ }^{6}$, Elizabeth Joan Barham ${ }^{7}$, Elisete Silva Pedrazzani ${ }^{8}$
}

${ }^{1}$ Resultados parciais da pesquisa "Tecnologia de cuidado para idosos com alterações cognitivas" financiada pela Financiadora de Estudos e Projetos do Ministério da Ciência e Tecnologia e pelo Conselho Nacional de Desenvolvimento Científico e Tecnológico (CNPq), no período de 2005-2007.

${ }^{2}$ Doutora em Educação. Professora Associado do Departamento de Enfermagem da Universidade Federal de São Carlos (UFSCar). São Paulo, Brasil.

${ }^{3}$ Doutor em Hidráulica e Saneamento. Professor do Departamento de Hidráulica e Saneamento da Escola de Engenharia de São Carlos (EESC) da Universidade de São Paulo (USP). Bolsista de Produtividade em Pesquisa do CNPq. São Paulo, Brasil.

${ }^{4}$ Doutor em Hidráulica e Saneamento. Pesquisador do Laboratório de Sistema de Informações Geográficas do Departamento de Hidráulica e Saneamento da EESC/USP. Bolsista de Desenvolvimento Tecnológico e Industrial do CNPq. São Paulo, Brasil.

${ }^{5}$ Graduando em Engenharia Ambiental. Pesquisador do Núcleo Integrado de Bacias Hidrográficas do Departamento de Hidráulica e Saneamento da EESC/USP. Bolsista de Iniciação Tecnológica e Industrial do CNPq. São Paulo, Brasil.

${ }^{6}$ Doutora em Serviço Social. Coordenadora do Programa do Idoso da Unidade Saúde Escola da UFSCar. São Paulo, Brasil.

${ }^{7}$ Doutora em Psicologia Social e Aplicado. Professora Associado do Departamento de Psicologia da UFSCar. São Paulo, Brasil.

${ }^{8}$ Doutora em Saúde Pública. Professora do Departamento de Enfermagem da UFSCar. São Paulo, Brasil.

PALAVRAS-CHAVE: Idoso. Demência. Saúde pública. Saúde da família. Sistemas de informação geográfica.

KEYWORDS: Aged. Dementia. Public health. Family health. Geographic information systems.

PALABRAS CLAVE: Anciano. Demencia. Salud pública. Salud de la familia. Sistemas de información geográfica.
RESUMO: Este trabalho descreve a construção de um sistema integrado de informações geográficas e de condições de saúde para idosos com demência, cadastrados nas Unidades de Saúde da Família de um município paulista. Trata-se de um estudo descritivo baseado em uma investigação quantitativa, desenvolvido no período de junho de 2006 a junho de 2007. Os passos metodológicos consistiram na criação de uma base de dados com atributos dos idosos, de um cadastro georeferenciado e de uma plataforma operacional para o armazenamento e gerenciamento do conjunto das informações. Foram visitados e avaliados 1.048 idosos, dos quais 275 apresentaram alterações cognitivas. A criação deste sistema permitiu visualizar a distribuição espacial dos idosos com demência, associar dados geográficos e de saúde, identificando fatores de risco e vulnerabilidade. A utilização de tecnologias de geoprocessamento mostrou-se importante no planejamento de ações de cuidado em gerontologia, contribuindo para a gestão de programas públicos de saúde.

ABSTRACT: This paper describes the construction of an information system, integrating geographic and health care data for elderly people with dementia who were registered in Family Health Units in a municipality in the State of São Paulo, Brazil. The study is descriptive, based on a quantitative investigation conducted between June, 2006 and June, 2007. The methodological steps included the preparation of a database, registering the attributes of the elderly; of a second, geo-referenced registry; and the development of an operational platform to link these two information sets. In-home evaluations were conducted with 1048 elderly, 275 of whom presented cognitive impairments. The creation of this system enables a visualization of the geographic distribution of elderly with dementia, the investigation of geographic and health data associations; and the identification of risk and vulnerability factors. As such, geo-processing technologies enable important new possibilities for planning care initiatives in gerontology, improving public health program management.

RESUMEN: En este trabajo se describe la construcción de un sistema integrado de informaciones geográficas y de las condiciones de salud para los ancianos con demencia, catastrados en Unidades de Salud de la Familia de un municipio paulista. Se trata de un estudio descriptivo con base en una investigación cuantitativa, desarrollado de junio de 2006 a junio de 2007. Los pasos metodológicos consistieron en la creación de una base de datos con los atributos de los ancianos, así como de de un catastro georeferenciado y de una plataforma operacional para el almacenamiento y gerencia del conjunto de las informaciones. Fueron visitados y evaluados 1.048 ancianos, de los cuales 275 presentaron alteraciones cognitivas. La creación de este sistema permitió visualizar la distribución espacial de los ancianos con demencia, asociar datos geográficos y de salud, identificando factores de riesgo y vulnerabilidad. La utilización de tecnologías de geoprocesamiento se mostró importante en el planeamiento de acciones de cuidado en gerontología, contribuyendo para la gestión de programas públicos de salud.
Sofia Cristina Iost Pavarini Endereço: R. Paraguai, 642 13.566-650 - Nova Estância, São Carlos, SP, Brasil. E-mail: sofia@power.ufscar.br emm@sc.usp.br
Artigo original: Pesquisa

Recebido em: 16 de julho de 2007 Aprovação final: 15 de janeiro de 2008 


\section{INTRODUÇÃO}

O envelhecimento populacional no Brasil vem ocorrendo de forma bastante acelerada. Associado a este fenômeno, a longevidade tem sido acompanhada da presença de doenças crônico-degenerativas, dentre as quais a demência merece especial atenção, uma vez que interfere na capacidade funcional dos idosos e constitui, atualmente, um grave problema de saúde mental e de saúde pública. ${ }^{1-3}$

As atenções de especialistas e pesquisadores voltam-se para a detecção precoce desta síndrome e para a criação de tecnologias de cuidado, com vistas à redução de seu impacto sobre a família e sobre o sistema público de saúde. Dentre as diferentes tecnologias demandadas para o atendimento ao usuário do serviço de saúde e sua família, destaca-se a utilização de tecnologias de geoprocessamento na gestão de programas públicos de saúde. A inclusão de informações geográficas significa a captação de dados sobre a localização do idoso no espaço e no tempo, em diferentes épocas, dias e horários. Integrar esses dados com informações sobre as condições de saúde e fatores de risco associados parece permitir importantes avanços no planejamento de ações de cuidado junto a idosos. Mais especificamente, pode auxiliar na localização de idosos com demência, que podem apresentar como um dos sintomas a desorientação têmporo-espacial. Desta forma, o desenvolvimento de metodologias e ferramental tecnológico, visando uma maior eficiência na gestão dos programas públicos de atendimento a esse segmento, ganhará um avanço significativo com a integração de um Sistema de Informações Geográficas (SIG) com dados de saúde.

Valendo-se do ferramental disponibilizado por meio das plataformas doSIG, é possível realizar o tratamento matemático de dados geográficos, bem como armazenar informações relacionadas à geometria e aos atributos dos dados georeferenciados (localizados sobre a superfície terrestre e representados numa determinada projeção cartográfica). ${ }^{4-5}$ Estas ferramentas podem ser incorporadas aos conhecimentos da área de saúde, tais como dados epidemiológicos e, assim, contribuírem para um maior reconhecimento da realidade local.

A informação geográfica apresenta uma natureza dual: possui uma localização ("onde", expressa como coordenadas em um espaço geográfico) e atributos descritivos ("o quê", que podem ser representados num banco de dados convencional). Sendo assim, sistemas de informação geográfica são empregados, principalmente, de três formas:
1) do ponto de vista cartográfico, para a geração e visualização de dados espaciais e produção de mapas; 2 ) do ponto de vista da base de dados, para o armazenamento e recuperação da informação espacial; e 3) do ponto de vista da análise espacial (por exemplo, identificação de regiões da cidade com alta concentração de idosos com demência), como suporte para a análise de fenômenos envolvendo a saúde dos idosos, por meio da inclusão das informações espaciais valendo-se de sua capacidade analítica e de modelagem.

A tecnologia do SIG está presente em diferentes áreas do conhecimento, o que lhe confere um caráter essencialmente transdisciplinar. $\mathrm{Na}$ área de saúde, o SIG tem sido classicamente aplicado no campo da epidemiologia, em investigações que procuram associar a distribuição de doenças e agravos em coletividades humanas. ${ }^{6}$

A identificação de fatores de risco para a saúde bucal, por exemplo, é facilitada pelo uso do SIG. Autores discutem a utilização do SIG a partir de uma série de aplicações nacionais e internacionais descritas na literatura, que incluem desde uma avaliação espacial da experiência de traumas dentários na região metropolitana de Curitiba - PR, até o mapeamento das ocorrências de cárie e sua correlação com a distribuição de água à população no País de Gales. A conclusão é de que há necessidade de maior desenvolvimento do referencial teórico quanto às metodologias a serem aplicadas, associado à disponibilidade de informações georeferenciadas em saúde bucal, que devem ser consideradas para a viabilização das aplicações do SIG na área de saúde.?

Além disso, o SIG possibilita melhorias na eficiência do uso de recursos públicos, porque permite cruzar dados sobre número de casos e localização das pessoas, para determinar como organizar o atendimento das mesmas. Um estudo realizado em Olinda - PE analisa a distribuição espacial da demanda por atendimento a casos de hanseníase, a fim de auxiliar na delimitação de áreas de domínio das unidades de saúde (associadas ao território de origem da população atendida). Neste caso, a integração do geoprocessamento ao sistema público de saúde possibilitou, segundo os autores, identificar elementos para a racionalização dos recursos e adequação dos serviços prestados nas Unidades de Saúde da Família (USFs) do município estudado. ${ }^{8}$

O SIG também tem se mostrado útil em aplicações de vigilância epidemiológica e entomológica. Com o objetivo de desenvolver um modelo 
de prevenção da transmissão de dengue em áreas urbanas, pesquisadores utilizaram técnicas de geoprocessamento de dados de saúde para localizar com precisão os casos da doença e a presença do vetor em Porto Alegre - RS. Os autores enfatizam a importância de integrar dados relacionados à intensidade de transmissão e à vulnerabilidade ao vetor, para o planejamento de ações de controle. ${ }^{9}$

No mesmo sentido, um estudo realizado em Ribeirão Preto - SP apresentou uma abordagem simples para distribuição espacial de casos de tuberculose neste município. Com base na espacialização dos casos da doença, os autores destacam as vantagens obtidas pela aplicação de ferramentas de geoprocessamento para o estabelecimento de conexões entre saúde e ambiente. ${ }^{10}$

Percebe-se que a demanda pela utilização, em questões de saúde pública, de plataformas computacionais aplicadas ao gerenciamento de informações sobre uma determinada base espacial vem aumentando consideravelmente no Brasil, acompanhando a evolução tecnológica e científica experimentada no campo do geoprocessamento.

Este trabalho teve por objetivo a construção de um sistema integrado de informações geográficas e de condições de saúde para idosos com demência, cadastrados nas USFs de um município paulista.

\section{METODOLOGIA}

Trata-se de um estudo descritivo baseado nos pressupostos do método quantitativo de investigação. Todos os princípios éticos que regem pesquisas com seres humanos foram respeitados. O projeto teve início após a aprovação pelo Comitê de Ética em Pesquisa da Universidade Federal de São Carlos, de Parecer No 055/ 2006.

O desenvolvimento do trabalho foi dado por aplicações realizadas em ambiente de Sistemas de Informações Geográficas (SIG). Sendo assim, o sistema englobou três elementos distintos: 1) uma base de dados de atributos, estabelecida pela identificação do idoso e pelo registro de informações sobre diferentes aspectos de suas condições de saúde e contexto de vida; 2) uma base georeferenciada de dados espaciais, que continha as informações espaciais mais relevantes para o objetivo do projeto, isto é, que permitiam uma rápida localização do idoso (por exemplo, logradouros, quadras e lotes); e 3) uma plataforma operacional que permitia a integração dos dois bancos de dados e a inserção do sistema no gerenciamento dos programas municipais de saúde.

\section{Base de dados de atributos}

Os dados de atributos foram coletados no domicílio dos idosos cadastrados, nas dez USFs do Município de São Carlos - SP. Foram consideradas pessoas com mais de 60 anos no dia primeiro de janeiro de 2006. Foram visitados e avaliados 1.048 idosos. Os dados foram coletados após treinamento prévio dos entrevistadores no período de junho de 2006 a junho de 2007.

O banco de dados de atributos era composto por dados sócio-demográficos (nome, idade, sexo, escolaridade, renda individual e familiar), localização (endereço e bairro), condições de moradia (tipo de habitação e condições de saneamento), estrutura e composição familiar (relação de parentesco com o idoso, pessoas que compõem a família, idade e sexo dos familiares), condições de saúde dos idosos (patologias referidas; rastreamento para depressão, usando a Escala de Depressão Geriátrica; rastreamento das alterações cognitivas, utilizando o Mini Exame do Estado Mental) e avaliação do desempenho dos idosos nas atividades básicas e instrumentais de vida diária, com a aplicação do Índice de Katz e Questionário de Pfeffer. ${ }^{11}$ Estes dados são exemplos de indicadores de risco e vulnerabilidade que devem ser considerados em programas de atendimento à população idosa com demência.

\section{Base georeferenciada}

Para a base georeferenciada foi utilizada a base cartográfica disponibilizada pela Secretaria Municipal da Fazenda e pela Secretaria Municipal de Habitação e Desenvolvimento Urbano do Município de São Carlos - SP, respeitando-se todos os princípios éticos dessa utilização. Esta base incluiu logradouros georeferenciados, bem como espaços públicos de interesse (por exemplo, ruas, avenidas, praças e serviços comunitários). Todas as variáveis de interesse foram projetadas na base cadastral do município. Para focalizar questões envolvendo a demência, a base foi composta apenas por dados dos idosos com indicação de alterações cognitivas ( $\mathrm{N}=275)$, segundo os resultados obtidos com os instrumentos da avaliação de atributos.

\section{Plataforma operacional}

Para a plataforma operacional, foram integrados os dados de atributos dos idosos com alterações cognitivas com a base de dados cadastrais georeferenciada. A integração foi possibilitada, no âmbito do geoprocessamento, por um conjunto 
de ferramentas disponibilizadas em softwares do SIG, que permitem consultar o banco de dados por meio da integração entre os dados geográficos e banco de dados de atributos.

A escolha do aplicativo do SIG utilizado nesta pesquisa envolveu os seguintes fatores: a) nível de confiabilidade e facilidade de acesso ao responsável pelo desenvolvimento do aplicativo; b) grau de desenvolvimento da versão disponível; c) facilidade de atualização futura; d) idioma do software e de sua documentação, para interação livre com usuários nos programas de saúde; e) interface amigável com o usuário; eff) facilidade no aprendizado de seu uso para agentes de saúde.

Com base nos critérios elencados e a partir da experiência dos autores com a operação de alguns softwares de geoprocessamento, optou-se por trabalhar com o aplicativo SPRING versão 4.3 , da Divisão de Processamento de Imagens, do Instituto Nacional de Pesquisas Espaciais. Este aplicativo permitiu procedimentos de análise e consulta espacial, por meio da ligação entre dados geográficos e dados de atributo a partir de um Sistema Gerenciador de Banco de Dados Relacional, em que os componentes espacial e descritivo dos objetos geográficos foram armazenados separadamente. Os atributos foram armazenados em bancos de dados convencionais, na forma de tabelas, e os dados espaciais foram tratados por um sistema georeferenciado de objetos. A conexão entre ambos foi feita por meio de identificadores numéricos que associavam o objeto geográfico ao campo correspondente na base de dados tabular. ${ }^{12}$

A fim de facilitar e dinamizar a utilização do sistema desenvolvido, a base de informações geográficas foi elaborada já levando em consideração o território das Unidades de Saúde vinculadas ao Programa de Saúde da Família (PSF) municipal, conforme divisão territorial adotada pela Secretaria Municipal de Saúde (Figura 1).

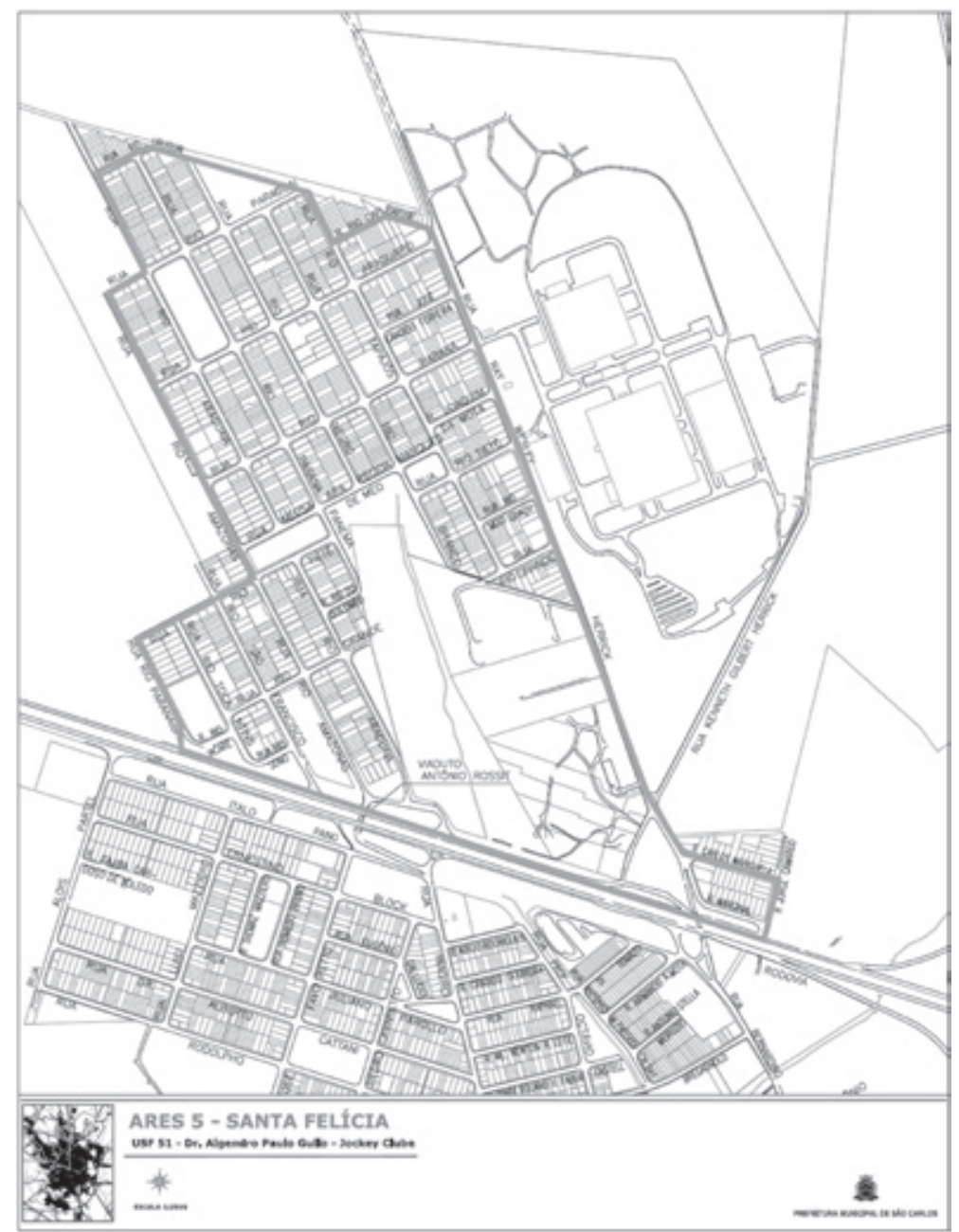

Figura 1 - Território de uma das USFs conforme estabelecido pela Secretaria Municipal de Saúde. São Carlos - SP, 2007. 


\section{RESULTADOS E DISCUSSÃO}

A título de exemplo, a Figura 2 apresenta os dados da plataforma de uma Unidade de Saúde da Família (USF) estudada. A figura mostra como o sistema é visualizado, pela espacialização dos elementos de interesse como a localização da USF, a malha viária e a localização dos idosos.

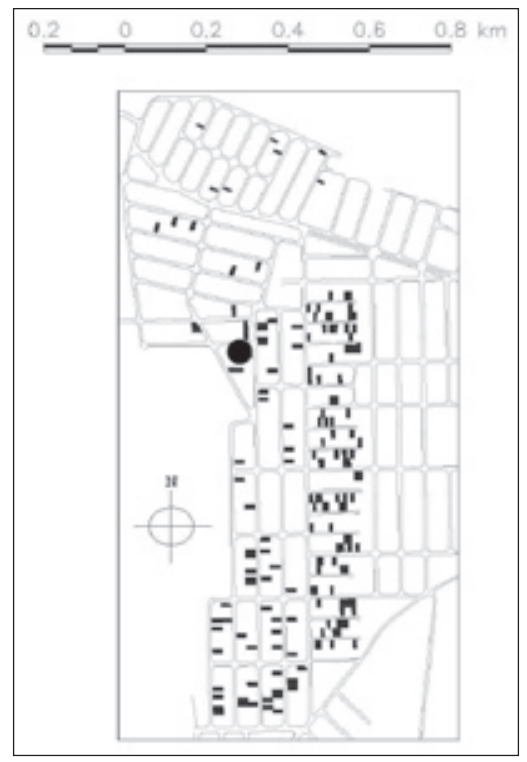

Figura 2 - Distribuição espacial dos idosos com alterações cognitivas de uma USF (indicada por um círculo). São Carlos - SP, 2007.
Uma primeira facilidade relacionada à aplicação do geoprocessamento refere-se à visualização da distribuição da demanda espacial (como ilustrado pela Figura 2), uma vez que o acompanhamento dos idosos pelos profissionais da área da saúde é realizado prioritariamente em suas residências. Essa informação, por si só, permite à equipe de saúde estruturar seu plano de atendimento, em função de variáveis como o tempo de deslocamento até a casa do idoso, a distância percorrida pelos agentes de saúde, entre outras.

A associação de dados espaciais e dados que descrevem as condições de saúde dos idosos, conforme mostra a Figura 3, oferece outras possibilidades de exploração do ferramental do SIG, relacionadas à otimização do atendimento nas unidades de saúde. A possibilidade de realizar consultas aos dados integrados no sistema pela localização do idoso a ser visitado, por exemplo, permite um melhor planejamento do atendimento em si, pela caracterização prévia do usuário e seu local de moradia. O envolvimento de diferentes profissionais também torna-se mais efetivo, uma vez que é possível reunir informações de diferentes fontes e ao longo do tempo.

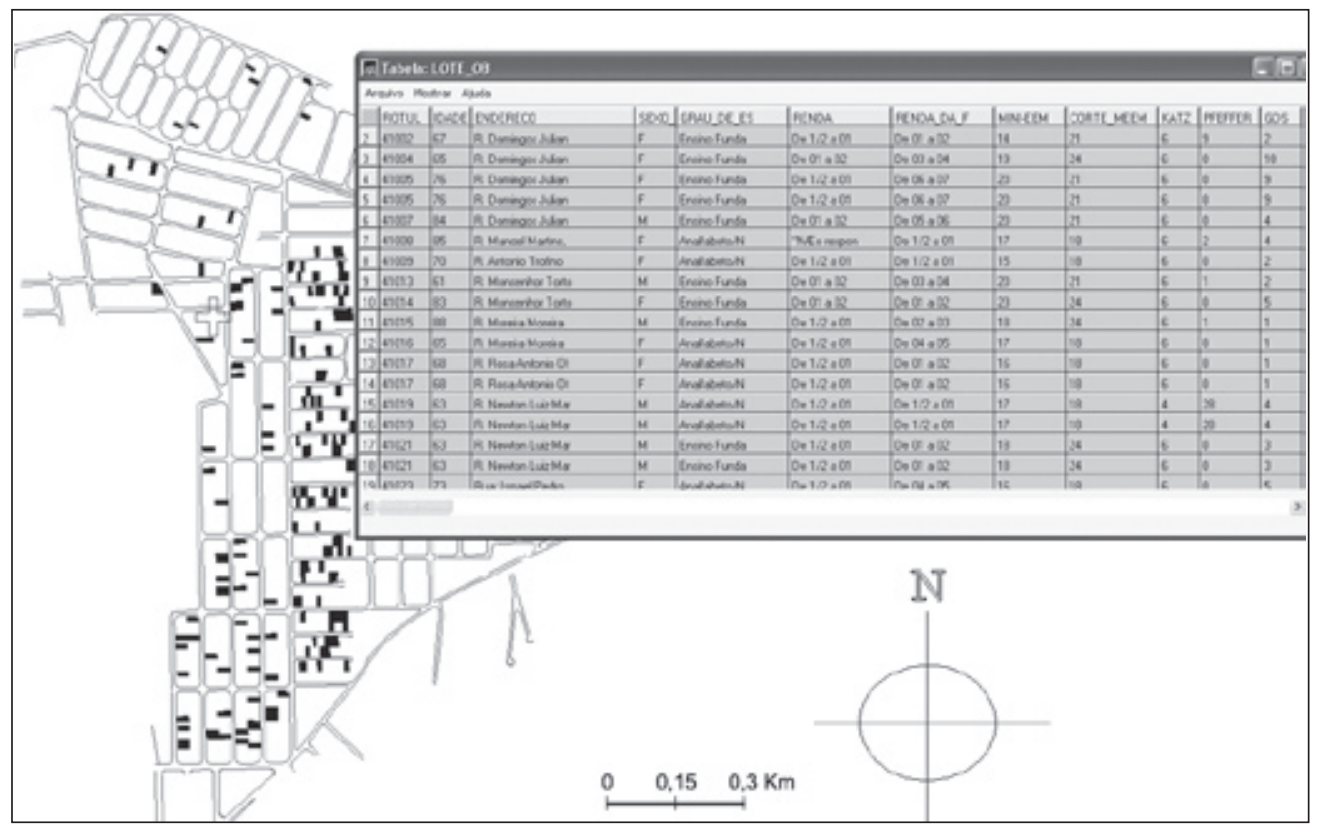

Figura 3 - Associação entre atributos (dados tabulares)* e dados espaciais (cartográficos) dos idosos com alterações cognitivas. São Carlos - SP, 2007.

* Respeitando a necessidade de proteger a privacidade de informações, os dados incluídos neste exemplo não contêm dados reais de identificação e de avaliação dos idosos. 
Certamente uma das principais contribuições relacionadas ao emprego do geoprocessamento para a gestão dos programas de atendimento a idosos, é a possibilidade de realização de consultas ao banco de dados, a partir das informações de localização geográfica (o sistema disponibiliza ao operador as informações armazenadas no banco de dados associados a uma determinada residência ou região geográfica), ou a partir dos atributos dos idosos (nesse caso, o sistema permite ao operador buscar a localização geográfica de idosos que apresentam determinadas características).

\section{Consulta por localização}

A consulta por localização (residência ou região geográfica) permite ao profissional acessar as informações armazenadas no banco de dados de atributos referentes a esta região. Trata-se de um procedimento extremamente simplificado quanto à sua operação, que depende apenas dos recursos disponibilizados pelo software a ser utilizado no desenvolvimento do produto.

A Figura 4 ilustra o procedimento de consulta. Ao clicar com o mouse sobre um determinado local ou região no mapa, (Rótulo $=41013)$, o sistema acessa o banco de dados e disponibiliza de modo automático as informações referentes ao polígono (local/região) selecionado. O procedimento pode ser efetuado para um conjunto de elementos, indistintamente. Para verificar, por exemplo, o número de casos de demência em determinada região da cidade, a delimitação da área de interesse permite consultar as informações sobre os idosos registrados no sistema, morando no bairro em questão.

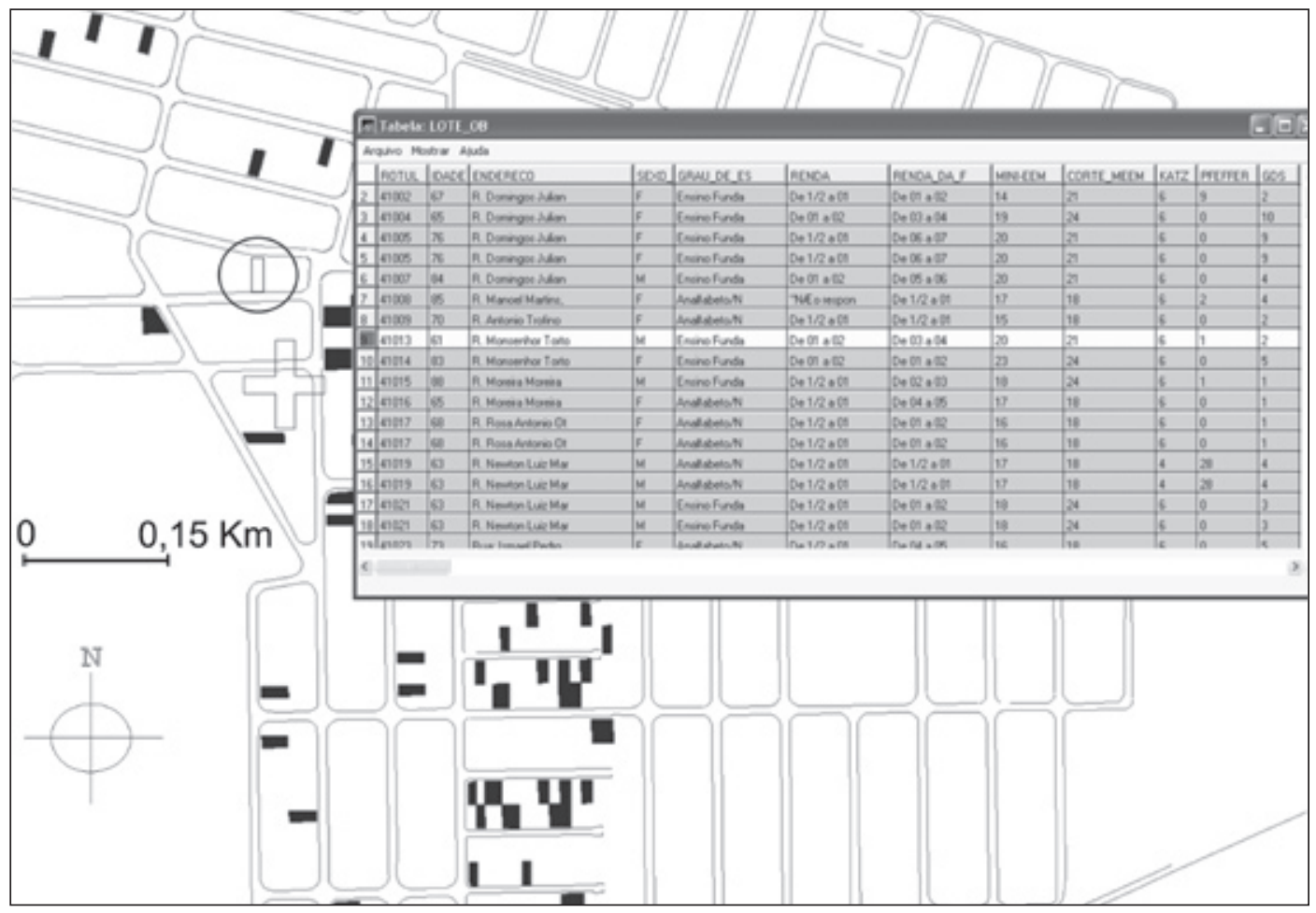

Figura 4 - Exemplo de aplicação do SIG para consulta ao banco de dados a partir da localização de um determinado elemento. São Carlos - SP, 2007.

\section{Consulta por atributo}

A consulta por atributo faz uma busca no sentido oposto: permite a localização dos idosos que atendem aos critérios de seleção (por exemplo, idosos com demência do sexo masculino), de modo a filtrar as informações conforme os objetivos. O siste$\mathrm{ma}$, ao aplicar um determinado filtro sobre o banco de dados, retorna ao leitor a localização dos idosos que se enquadram nos critérios estabelecidos. Seria possível, por exemplo, visualizar a distribuição geográfica de casos de demência avançada.

A Figura 5 mostra uma aplicação sobre o banco de dados de atributos para uma das unidades de saúde estudadas. Para encontrar a localização de 
idosos do sexo feminino com alterações cognitivas moderadas, o profissional estabelece o conjunto de critérios: mostre-me a localização, apenas, dos idosos do sexo feminino que obtiveram pelo menos 5 pontos a menos de sua nota de corte* no Mini Exame do Estado Mental (MEEM). Isso significa que o sistema deve encontrar no banco de dados aqueles idosos que satisfazem, simultaneamente, as seguintes condições:

$$
\begin{aligned}
& \text { [Campo “SEXO”] = F } \\
& \text { [Campo “CORTE_MEEM] - [Campo } \\
& \text { "MEEM"] } \geq 5
\end{aligned}
$$

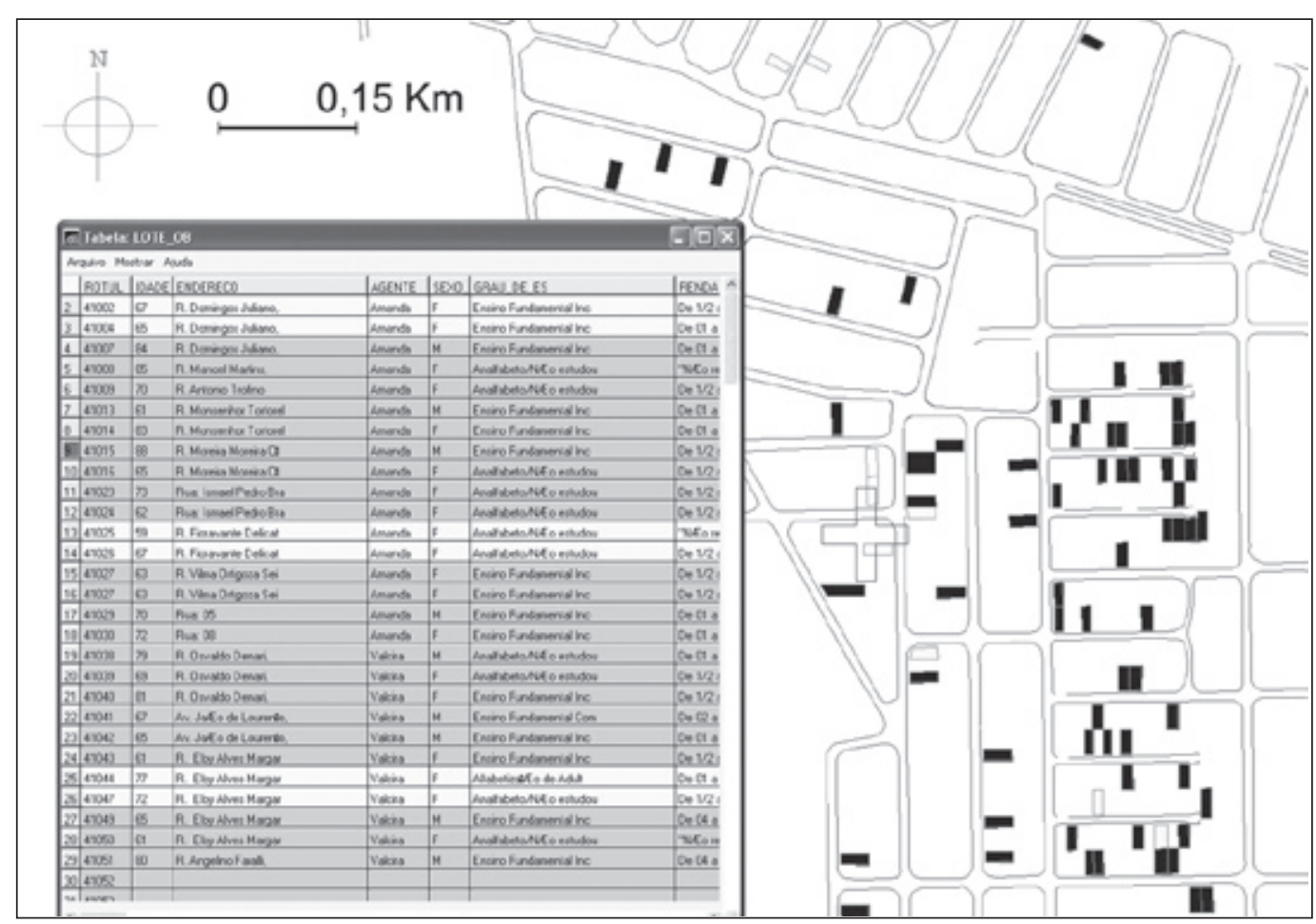

Figura 5 - Consulta por combinação de atributos - o sistema localiza idosos que se enquadram nos critérios estabelecidos. São Carlos - SP, 2007.

Este estudo limitou-se a descrever a construção de um sistema integrando o georeferenciamento e as condições de saúde dos idosos com demência e ilustrar o potencial deste sistema. Usando esta tecnologia será possível desenvolver pesquisas futuras para a análise das relações entre fatores espaciais e temporais com as condições de saúde dos idosos avaliados. A aplicação do SIG em investigações em saúde oferece importantes possibilidades de novas análises e é uma ferramenta essencial para a integração de informações sobre a saúde e o ambiente. ${ }^{10}$

Autores têm aprofundado o uso do SIG na saúde pública em geral. ${ }^{10,13-16}$ Outros autores discutem a demanda para o uso das ferramentas analíticas do $\mathrm{SIG}^{17}$ ou sugerem a ampliação da coleta de dados para incluir novos indicadores de saúde pública que permitem a maior integração de dados de saúde com dados ambientais. ${ }^{18}$

No caso dos idosos com demência, o SIG permitirá investigar associações entre a localização dos idosos e fatores de risco e vulnerabilidade. A título de exemplo, seria instrutivo investigar a relação entre a distribuição espacial dos idosos com maior comprometimento cognitivo (portanto, com maior necessidade de cuidados), e indicadores dos recursos sociais e de apoio disponíveis em cada bairro (incluindo os recursos familiares, o gênero dos cuidadores, a renda familiar) entre outras possibilidades de relação.

A plataforma criada neste estudo permitirá, também, a inclusão de outras variáveis impor-

* A nota de corte no MEEM depende da escolaridade do idoso. 
tantes sobre as condições de saúde dos idosos além da demência, como hipertensão, diabetes, osteoporose, problemas emocionais e distúrbios comportamentais.

Considerando que este trabalho foi realizado no âmbito do PSF, seria desejável utilizar este novo recurso para analisar a relação entre diferentes aspectos do ambiente e as condições de saúde dos idosos e de seus familiares, como cônjuge, filhos, netos e outros.

Dado que a demência é um grave problema de saúde pública, a utilização de tecnologias de geoprocessamento nos serviços públicos de saúde é de extrema importância, pois permite uma análise da combinação de fatores de risco em um determinado local, que podem levar à maior fragilidade dos idosos. Estas análises certamente trarão contribuições significativas para o cuidado ao idoso com demência no município.

\section{CONCLUSÕES}

A integração de dados georeferenciados e o banco de dados de atributos dos idosos, permitiu a construção de um sistema para armazenamento e manipulação de dados referentes aos idosos sendo acompanhados pelas USFs do município estudado. Este sistema oferece aos gestores não apenas os dados das condições de saúde e de vida dos idosos, como os bancos de dados tradicionais, mas também a distribuição espacial dos mesmos, além da possibilidade de realização de análises cruzando questões de saúde com dados envolvendo questões espaciais e temporais.

A união de ferramentas consagradas no estudo da saúde com as tecnologias para análise de dados georeferenciados, também consagradas em sua área, aumenta sobremaneira as possibilidades de análise das variáveis de interesse, contribuindo para uma melhor gestão da saúde. Especificamente no âmbito municipal, a agregação de sistemas operacionais baseados em softwares livres permite acesso a esta nova tecnologia para uso nos programas públicos de saúde.

O geoprocessamento constitui um excelente instrumento para a melhoria de programas de atendimento a idosos, por meio do exame das características e localização dos indivíduos que integram tais programas. Nesse sentido, destacam-se como pontos positivos a possibilidade de atualização contínua dos dados (espaciais e de atributos) e sua imediata integração a uma plataforma operacional como a apresentada.
Assim, a utilização de tecnologias de geoprocessamento na gestão de programas públicos de saúde mostrou-se importante no planejamento de ações de cuidado em gerontologia e, certamente, permitirá a inclusão de novas e diferentes informações de saúde da população atendida pelo PSF do município.

\section{AGRADECIMENTOS}

Agradecemos o apoio financeiro da Financiadora de Estudos e Projetos do Ministério da Ciência e Tecnologia, os recursos aprovados através do Edital Tecnologia Assistida e bolsas de Iniciação Tecnológica e Industrial e Desenvolvimento Tecnológico e Industrial.

\section{REFERÊNCIAS}

1 Lebrão ML, Duarte YAO. Saúde, bem-estar e envelhecimento: o projeto Sabe do município de São Paulo, uma abordagem inicial. Brasília (DF): OPAS; 2003.

2 Luzardo AR, Gorini MIPC, Silva APS. Características de idosos com doença de Alzheimer e seus cuidadores: uma série de casos em um serviço de neurogeriatria. Texto Contexto Enferm. 2006 OutDez; 15 (4): 587-94.

3 Bottino CMC, Laks J, Blay SL. Demência e transtornos cognitivos em idosos. Rio de Janeiro (RJ): Guanabara Koogan; 2006.

4 Davis C, Fonseca F. Introdução aos sistemas de informação geográficos. [Curso de Especialização em Geoprocessamento]. Belo Horizonte (MG): UFMG/ Instituto de Geociências; 2001.

5 Eastman JR. IDRISI for windows user's guide version 32: introduction. Worcester (MA/USA): Clark University/Graduate School of Geography; 1999.

6 Barcellos C, Bastos FI. Geoprocessamento, ambiente e saúde: uma união possível? Cad. Saúde Pública. 1996 Jul-Set; 12 (3): 389-97.

7 Moreira RS, Nico LS, Tomita NE. A relação entre o espaço e a saúde bucal coletiva: por uma epidemiologia georeferenciada. Ciênc. Saúde Colet. 2007 Jan-Mar; 12 (1): 275-84.

8 Lapa TM, Albuquerque MFPM, Carvalho MS, Silveira Júnior JC. Análise da demanda de casos de hanseníase aos serviços de saúde através do uso de técnicas de análise espacial. Cad. Saúde Pública. 2006 Dez; 22 (12): 2575-83.

9 Barcellos C, Pustai AK, Weber MA, Brito MRV. Identificação de locais com potencial de transmissão de dengue em Porto Alegre através de técnicas de geoprocessamento. Rev. Soc. Bras. Med. Trop. 2005 Maio-Jun; 38 (3): 246-50. 
10 Hino P, Villa TCS, Sassaki CM, Nogueira JÁ, Santos CB. Geoprocessamento aplicado à área da saúde. Rev. Latino-Am. Enfermagem [on line]. 2006 Nov-Dez; 14 (6): 939-43 [acesso em 2007 Maio 12]. Disponível em: www.eerp.usp.br/rlae

11 Nitrini R, Caramelli P, Bottino CMC, Damasceno, BP, Brucki, SMD, Anghinah R. Diagnóstico de doença de Alzheimer no Brasil: critérios diagnósticos e exames complementares, recomendações do Departamento Científico de Neurologia Cognitiva e do Envelhecimento da Academia Brasileira de Neurologia. Arq. Neuro-psiquiatr. 2005 Sep; 63 (3-A): 713-9.

12 Lopes ESS, Ferreira HS. Tutorial 10 aulas SPRING 4.3. São José dos Campos (SP): Instituto Nacional de Pesquisas Espaciais; 2006.
13 Cromley EK. Gis and disease. Ann. Rev. Public Health. 2003 Jan; 24:7-24.

14 Ricketts TC. Geographical information systems and public health. Ann. Rev. Public Health. 2003 Jan; 24: 1-6.

15 Croner C. Public health, GIS and the internet. Ann. Rev. Public Health. 2003 Jan; 24: 57-82.

16 McLafferty SL. GIS and health care. Ann. Rev. Public Health. 2003 Jan; 24: 25-42.

17 Rushton G. Public health, GIS and spatial analytical tools. Ann. Rev. Public Health. 2003 Jan; 24: 43-56.

18 Etches V, Frank J, Di Ruggiero E, Manuel D. Measuring population health: a review of indicators. Ann. Rev. Public Health. 2006 Jan; 27: 29-55. 